\title{
78TH ANNUAL SASKATCHEWAN CHRISTMAS BIRD COUNT - 2019
}

Alan R. Smith

Box 154

Avonlea SK SOH OCO

alanrandi@sasktel.net

\section{The Counts}

Only 74 counts were received for the 2019-20 count, a most concerning decline over last year's 86 and the fewest since 1984. There are a number of possible reasons for this. Human demographics may play a role as aging rural populations and increasing urbanization mean that fewer 'ma and pa' counts are conducted in the hinterland. On the other hand, more and more people are involved in a fewer number of counts conducted in or near larger urban centers.

Another factor is technological, as I have the suspicion that I may have inadvertently deleted a small number of emailed counts. If you sent me a count that does not appear in this report, please send me a copy (hopefully you still have one) and I will ensure that your results will be included in our long-term database.

The bottom line is that while the total number of counts was down by 14 per cent, the number of observers was only down by four per cent; this supports the contention that urbanization is at least partially responsible for the decline in the overall number of counts conducted.

\section{The Weather}

Average minimum and maximum temperatures for the count period (with 2018-19 records in brackets) were -11 to $-6 C(-10$ to $-5 C)$, wind speeds 8 to $16 \mathrm{~km} / \mathrm{h}$ ( 8 to $19 \mathrm{~km} / \mathrm{h}$ ), and snow depths 5 to $12 \mathrm{~cm}$ ( 6 to
$14 \mathrm{~cm}$ ). As can be seen, there was little difference in these conditions from last year. One difference, however, was the prevalence of fog. Fog was reported on seven counts this year as opposed to three last year.

This may have hampered observers especially in the early morning hours.

\section{The Birds}

The 126,813 birds counted was higher than last winter's 108, 164 and slightly higher than the century average of 125,000 . This is partly due to a record high count of 33,735 Canada Geese on the Estevan count.

The 98 species recorded on count day was about average for the century, while the average number of species per count at 19.1 was the highest since 2012 with its record high of 19.4 .

For the third time in the past four years, Gardiner Dam won the crown for the most species at 45 . The runner-up was Saskatoon with 40 species.

\section{Population Trends}

Waterfowl were generally found in normal numbers, except for new provincial highs for Canada Geese and Common Goldeneye (Table 6).

Most raptors were generally at or below normal numbers this winter. Exceptions were the Northern Goshawk and Gyrfalcon, which staged minor incursions into the southern portion of the province. Twenty-eight goshawks were recorded on 20 counts compared to 16 on 14 counts last winter. Respective numbers for gyrs were nine birds on nine counts compared to only three on three counts in 2018-19.

After decades of growth, the range expansion for the introduced Eurasian Collared-Dove and House Finch has come nearly to a halt. The only new CBC localities were LoveTorch River for the dove and Estuary North for the finch.

Numbers of the subarctic-nesting Common Redpoll were down again with only 557 birds on 27 counts compared to 3,412 birds on 55 counts in 2018-19 and 14,307 on 78 counts in 2017-18.

Numbers of most other finches were either down or near normal in numbers. There were, however, a couple exceptions. In a rare reversal of the norm, the Red Crossbill was more common and widespread than the White-winged Crossbill with 92 birds on seven counts versus seven white-wings on two counts. Graycrowned Rosy-Finches were also more in evidence with 29 birds at six localities; this species is usually found in only one or two localities a year.

\section{Rarities}

The Pacific Loon was a new species for the $C B C$ bringing the all-time total to 192 species. A few other rarities of note were a seventh Double-crested Cormorant at Gardiner Dam and a $10^{\text {th }}$ record of Northern Cardinal at Prince Albert.

Note: for purposes of Saskatchewan Counts as published herein, the count period extends from 14 December to 5 January; Audubon counts include only those species recorded three days prior to 
and after the count day.

\section{Count Areas and Participants (names of compilers are in italics)}

1. ARCHERWILL. Sharron Carlson, Gerald Hiron, Susan Hiron, Audrey Hnetka, Perry Hnetka, Elaine Hughes, Dorothy Klettberg, Willie Klettberg, Annette Kozak.

2. AVONLEA. Randi Edmonds, Alan Smith, Blaine Sudom, Graham Thomson.

3. BALGONIE. Jared Clarke, Jeff Gamble, Ken Feltin, Keith Hay, Fran Kerbs, Maureen Lee, Kristen Martin, Brett Quiring, Dan Sawatzky, Mary Worel, Doreen Yurkoski.

4. BIGGAR. Dale Booth, Mary-Jo Danychuk, Ryan Dudragne, Sheila Jezowski, Murray Newton, Mark Pickett, Monika Slowski, Guy Wapple, Marguerite Wapple, Rob Wapple.

5. BIRCH HILLS. Margaret Mareschal, Maurice Mareschal.

6. BORDEN-RADISSON. Jade Allard, Greg Fenty, Kyron Giroux, Ron Jensen, Nick Saunders, Stan Shadick, Joe Stookey, Guy Wapple.

7. BROADVIEW. Barb Weidl, Don Weidl.

8. CANDLE LAKE. Marie Braaten, Gwen Klebeck, Bea Kolbialko, John Lundgren, Vicki St. Germaine, Warren St. Germaine.

9. CATER: Beverly Beland, Orval Beland.

10. CHATSWORTH. Herb Cross, Jean Cross, Charles Dyck, Donna Dyck, Robert Mess, Wyonna Mess, George Murray, Laurie Murray, Kerri Rooke, Stewart Rooke, Carol Tangedal, Tony
Tangedal.

11. CHURCHBRIDGE A. Elin Johnson, Ron Johnson.

\section{CHURCHBRIDGE B. Dennis} Petracek.

\section{CLARK'S CROSSING. Dale}

Booth, Dave Cook, Louise Cook, Jess Cosentino, Ana Diaz, Lorne Duczek, Shelly Fisher, Daniel Giesbrecht, Glen Giesbrecht, Bob Godwin, Dawn Hall, Marilyn Haskins, Ron Jensen, Robert Johanson, Carson Kearns, Gwen Klypak, Heney Klypak, LeeAnn Latremouille, Brian McGill, Janine McManus, Joe Monahan, Lynn Oliphant, John Patterson, Al Scholz, Jan Shadick, Stan Shadick, Rhonda Shewfelt, Marten Stoffel, Stookey, Guy Wapple, Diane Wells, Michael Williams.

\section{CRAVEN. Hiroyuki Aoki, Brian} Armstrong, Stephane Canevet, Joel Cherry, Blanche Cooper, Jim Cummings, Stephen Davis, Elaine Ehman, Chris Harris, Trevor Herriot, Louise Holloway, Phil Holloway, Bonnie Huculak, Laurie Koepke, Lauretta Lane, Bob Luterbach, Barbara Mader, Amelia McDonald, Jaret McDonald, Annie McLeod, Barry Mitschke, Dave Phillips, Rhonda Phillips, Curtis Pollock, Brett Quiring, Susan Rollins, Dan Sawatzky, Nick Selinger, Jeanette Taylor, Robb Taylor, Rand Teed.

\section{CROOKED LAKE. Boyd Metzler.}

16. CROOKED RIVER. Karl Mehler, Margaret Mehler, Morley Mehler.

17. CYPRESS HILLS PROVINCIAL PARK (Centre Block). Caitlin Arnal, Dwight Dobson, Nancy Dobson, David Larson, Margaret Larson, Melody Nagel-Hisey, Alison Nagy, Royce Pettyjohn, Darcy Perrin, Jerry Perrin, Annette Schwazer, Brenda Sunde, Melvin Sunde, Aaron Sulz, Jill Sulz.
18. DENHOLM. Beverly Beland, Gerard Beland, Linda Beland, Orval Beland.

19. DORINTOSH. Vicky Pryor, Joe Twidale, Lorraine Twidale, Tim Wahl.

20. DUVAL. Greg Cutchey, Lloyd Saul.

21 E. B. CAMPBELL DAM. Bert Dalziel, Joan Dalziel, Sara Dalziel, Richard Douslin, Ryan Dudragne, Stan Shadick, Bill Weighill, Clara Weighill.

22. EASTEND. Loraine Armstrong, Roxie Binkley, Duane Bristow, Kevin Bristow, Cynthia Fehr, Robert Gebhardt, June Higgins, Joan Hodgson, Harvey Johnson, Heidi Topham, Jack Wilkinson.

23. EBENEZER. Ronan Koziy, Kenn Wood, Sarah Wood

24. ESTEVAN. Barry Dies, Marilyn Dies, Gary Leslie, Craig Palmer, Larry Preddy, Reid Walton, Rose Walton, Guy Wapple, Sandra Wapple.

25. ESTUARY NORTH. Cathy Cocks, Dean Francis, Fran Francis.

26. FENTON. Carman Dodge.

27. FLORAL. Guy Wapple.

28. FORT QU'APPELLE. Jean Ashcroft, Peter Ashcroft, Shirley Hart Carol Horsman, Don Horsman, Stewart Klyne, Jack Lowe, Marg Lowe, Alan Mlazgar, Denise Mlazgar, Keith Stephens, Kim Stephens, Ted Stevenson, Colette Stushnoff, Richard Stushnoff, Dave Sutherland.

29. GARDINER DAM. Ryan Dudragne, Greg Fenty, Maxine Forsberg, Ron Jensen, Nick Saunders, Stan Shadick, Marten Stoffel, Joe Stookey, Guy Wapple. 
30. GOOD SPIRIT LAKE. Joyce Anaka Dorothy Riesz, Ray Riesz.

31. GRAYSON. Jack Carrigan, Carina Helm, Charles Helm, Daniel Helm, Linda Helm, Jeanette Zimmer, Karl Zimmer.

32. GRENFELL. Ethel Reiger, Barb Weidl, Don Weidl.

33. HARRIS. Ryan Dudragne, Ron Jensen, Bruce Trapp, Guy Wapple, Rob Wapple.

\section{HAZLET. Ryan Dudragne.}

35. INDIAN HEAD. Ron Chambers, Mike Duran, Irvin Escott, David Gehl, Roberta Gehl, Ed Haid, Edith Haid, Jim Jinks, Linda Jinks, Linda Kort, Dan Loran, Bruce Neil, Dora Nichols, Laurie Norman, Laura Poppy, Brian Scott, Glenn Scott, Lorne Scott, Fred Skinner, C. Skinner, Joan Taylor, Donna Thompson, Rebeca Ward, Elaine Williamson.

36. KENASTON. Doug Beckie, P. Lawrence Beckie, Tyler Beckie.

37. KENOSEE LAKE. Boyd Metzler, John Pollock.

38. KETCHEN NORTH. Dallas Fairburn.

39. KINLOCH. Don Forbes, Carter Haroldson, Robert Haroldson, Ashley Messner, Doreen Wickstrom.

40. KYLE. Janine McManus, Yvonne Nelson, Marten Stoffel, Dan Zazelenchuk.

41. LA RONGE. Doug Bagwell, Linda Mikolayenko, Sid Robinson, John Schisler, Jan Shewchuk.
42. LOVE - TORCH RIVER. Bert Dalziel, Joan Dalziel, Sara Dalziel, Scott Edwards, Andrea Fisher, Harold Fisher, Taren Fisher, Adrik Kurbis, Coulter Kurbis, Duane Kurbis, Renee Kurbis, Eileen L'Heureux, Audrey Schrader, Eric Schrader, Leonard Turtle.

43. LUSELAND. Maxine Butler, Kelly Cotter, Estelle Finley, Graeme Finley, Kim Finley, Liam Finley, Valerie Finley.

44. MELFORT. Bert Dalziel, Joan Dalziel, Gordon Dodds, Susan Dodds, Emily Eskowich, Kim Eskowich, Wendy Eskowich, Darlene Thompson.

45. MOOSE JAW. Chris Harris, Bob Luterbach, Jeff Mander, Brett Quiring, Ed Rodger, Dan Sawatzky.

46. MOOSE MOUNTAIN. Bill Fletcher, Dorothy Fletcher, Doyle Thomas, Val Thomas.

47. MORSE. Elizabeth Enns, Jon Enns, Noel Enns, Stella Enns, Mike Francis, Randy McCulloch, Joel Priebe, Ken Priebe, Myrna Priebe, Lori Wilson.

48. NICOLLE FLATS. Gale Fennell, Dale Hjertaas, Fran Kerbs, Jeff Mander, Mary Worel.

49. NIPAWIN. Nancy Budd, Dave Christiansen, Joyce Christiansen, Bert Dalziel, Joan Dalziel, Betty Dolman, Rick Douslin, Patti Gaertner, Carolyn Grimm, Jennette LeCuyer, Jeri McCleary, Peter McCleary, Fred Olfert, Doug Phillips, Shirley Phillips, Leonard Turtle.

50. NISBET FOREST, WEST. Kim Clark, Shamara Clark, Suzanne Clark.

51. ODESSA. Arden Curts, Denise

Curts, Denny Curts.
52. OUTLOOK. Graham Thomson.

53. PIKE LAKE. Hadi Asgari, Nick Belliveau, Sara Brison, Donna Bruce, Dave Cook, Louise Cook, Lorne Duczek, Ryan Dudragne, Mary Jane Eley, Joan Feather, Alex Fisher, Bob Godwin, Janet Hill, Bob Howe, Irene Howe, Shirley Humphries, Greg Hutchings, Erin Lang, LeeAnn Latremouille, Greg Lawrence, Marilyn Leuty, Rod Luety, Audrey MacKenzie, Bill MacKenzie, Janine McManus, Kathy Meeres, Merhnaz Mikhchian, Joe Monahan, John Patterson, Marc Sabourin, Monica Salles, Nick Saunders, Nicola Schaefer, Joe Stookey, Louisa Stuglin, Stephan Stuglin, Moriah Tanguay, Guy Wapple, Adrian Werner, Dwight Young, Nancy Young.

\section{PONTEIX. Ryan Dudragne.}

55. PRINCE ALBERT. Doug Braaten, Marie Braaten, Ffion Cassidy, Kim Clark, Shamara Clark, Bert Dalziel, Joan Dalziel, Carman Dodge, Harold Fisher, Shelley Fisher, Ham Greenwood, Dale Jefferson, Gwen Klebek, Bea Kobialko, Les Love, John Lundgren, Catherine Miller, Lorna Mumm, Shannon Poppy, Christine Rye, John Rye, Vicki St. Germaine, Warren St. Germaine, Don Weidl, Lusi Wells.

56. QU'APPELLE. Jean Ashcroft, Peter Ashcroft, Cory Bennett, Christine Blair, Colette Stushnoff, Richard Stushnoff, Frank Veresh.

\section{QU'APPELLE VALLEY DAM.} Ryan Dudragne, Robert Johanson, LeeAnn Latremouille, Jan Shadick, Stan Shadick, Carl Siemens, Hollyce Siemens, Marten Stoffel, Guy Wapple, Michael Williams. 
58. REGINA. Ingrid Alesich, Brian Armstrong, Lionel Bonneville, Stephane Canevet, Janet Canwood, Joel Cherry, Lolamae Crawley, Ron Crawley, Derek Donald, Jim Elliott, Jaquie Fauth, Phyl Fauth, Mary Field, Terry Ford, Shirley Friel, Tammi Gillies, Avery Gillies, Brendan Graham, Chris Harris, Keith Hay, Norm Henderson, Trevor Herriot, Dale Hjertaas, Bruce Holmes, Kara Karst, Jim Karst, Fran Kerbs, Andre Kroeger, Nikky Kroeger, Assol Kubeisinova, Maureen Lee, Bob Luterbach, Lauren Mang, Kim Mann, Val Mann, Annie McLeod, Wayne Pepper, Brett Quiring, Chris Ratch, Ed Rodger, Keith Roney, Dan Sawatzky, Nick Selinger, Margaret Skeel, Brian Sterenberg, Frank Switzer, Mary Switzer, Hanna Walczykowski.

\section{ROSCOMMON S.D. Bernice}

Althouse, Jim Althouse, Kate

Althouse, Ruby Finnie, Brian Irving, Sophie Jankowski, Joan Lillibo, Dianne Sloan, Graham Sloan, Marguerite Sloan.

60. ROULEAU. Stuart Anderson, Allen McGratten, Noreen McGratten, Patricia Sterzuk.

61. ROUND LAKE (Qu'Appelle Valley). Boyd Metzler, Gary Tinnish, Mary Ward, Pat Ward.

\section{SALTCOATS. Arden Bradford,} Donna Bradford, Olga Brygider, Walter Brigadier, Len Cameron, Muriel Cameron, Dave Herron, Gloria Herron, Gerri Knudsen, Ron Knudsen, Fern McKay, Les Pearson, Shirley Pearson, G. Rathgaber, Monique Smith, Ken Trowell, Merdell Trowell, Val Trowell, Earl Upshall, Verda Upshall, Joan Wilson, Rob Wilson.

\section{SASKATCHEWAN LANDING} PROVINCIAL PARK. Ryan Dudragne, Janine McManus, Glen Pederson, Marten Stoffel, Dan Zazelenchuk.
64. SASKATCHEWAN RIVER FORKS. Carman Dodge, Don Weidl.

65. SASKATOON. Joanne Adams, Eveline Boudreau, Delores Burkhart, Yvonne Cuttle, Glenn Dougan, Lorne Duczek, Ryan Dudragne, Joan Feather, Leslie Fell, Greg Fenty, Jennifer Fenty, David Forbes, Martin Gerard, Bob Godwin, Mike Gollop, Donna Goodridge, Jim Goodridge, Jeff Harder, Jacob Henderson, Lorie Henderson, Janet Hill, Terri Jackson, Richard Kerbes, Gordon Koshinsky, Margaret Koshinsky, Erin Lang, Don Loran, Janet Loran, Priscilla Mah, Valerie Martz, Janine McManus, Bob McNaughton, Kathy Meeres, Scott Mitchell, John Patterson, Ava Paul, Gerald Paul, Jim Paul, Lisa Rohachuk, Marc Sabourin, Craig Salisbury, Lorriene Salisbury, Scott Saretsky, Nick Saunders, Jan Shadick, Stan Shadick, Aaron Shingoose, Garry Shurry, Todd Shurry, Kathlin Simpkins, Janice Solem, Jennifer Solem, Barb Sprigings, John Steel, Marten Stoffel, Margo Taylor, Phil Taylor, Jack Waisanen, Guy Wapple, Cathy Watts, Hamish Watts, Olive Watts, Trent Watts, Diane Wells, Jim Wells, George West, Helen Wilkins, Bev Will, Michael Williams, Margaret Woloshyn, Dan Zazelenchuk, Norman Zlotkin.

66. SHAMROCK. Hugh Henry, Iris McNeill, Darwin Menke, Joel Priebe, Ken Priebe, Myrna Priebe, Clinton Rud, Lori Wilson.

\section{SHELL LAKE. Ryan Dudragne,} Carole Martin, Blair Miller, Sharon Miller, Nick Saunders, Kay Willson, Phil Willson.

68. SNOWDEN. Sonja Fidyk, Ed Hagel, Irene Hagel, Doreen Long, Linda Patton, George Pickett, Jack Pickett, Karen Priestley, Valerie Rien.
69. SWIFT CURRENT. Jacquie Bolton, Stacy Bolton, Norris Currie, Gordon Dowie, Laurent Dudragne, Mary Ann Dudragne, Arnie Ens, Lorne Fast, Dave Green, Esther Green, Katie Hagman, Norma Hain, Hugh Henry, Leonard Howes, Lois Howes, Michelle Hubbard, Vera Lynn Knipful, Nicole Kuyek, Connie Lendrum, Dot Letkeman, Robert Moroz, Janet Payne, Arden Pierce, June Roy, Harold Steppuhn, Sue Steppuhn, Irene Stinson, Kae Watters.

70. TURTLEFORD. Hank deGraaf, Marlene deGraaf, Richard Hughes, Miles Johnson, Brent Keen, Harry Lake, Louise Lundberg, Ron Perkins, Lisa Richards, Richard Roney, Mark Seabrook, Dorothy Textor, Margaret Uhlig, Marilyn Whelan.

71. WEYBURN. Glen Fleming, Millie Fleming, Dale Huff, Sandy Huff, Murray Keefe, Charlotte Payak, Don Payak, Janice Phillips, Garnet Schultz, Lina Schultz, Joe Stephaniuk, Doyle Thomas, Tanis Thomas, Val Thomas, Kim Thorson, Myrt Thorson, John Whitell.

72. WHITE BEAR. Martin Gerrard, Yvonne Nelson, Deb Peterson, Marten Stoffel, Dan Zazelenchuk.

73. WHITEWOOD. Ken Aldous, Cindy Ashfield, Joe Ashfield, Paul Ashfield, Kerri Bachtold, Joyce Kydd, Sarah Mambourg, Boyd Metzler, Margaret Niemenen, Paul Niemenen, Brenda Pollock, John Pollock, Tony Saltasuk, Carole Sawatsky, Doug Shepherd, Dawn Vennard, Diane Veresh, Pat Ward.

74. WINGARD. Rebecca Beam. 
TABLE 1. Weather and Snow Cover.

\begin{tabular}{|c|c|c|c|c|c|c|c|c|c|}
\hline LOCALITY & DATE & $\begin{array}{c}\text { MIN } \\
\text { TEMP } \\
\left({ }^{\circ} \mathrm{C}\right)\end{array}$ & $\begin{array}{c}\text { MAX } \\
\text { TEMP } \\
\left({ }^{\circ} \mathrm{C}\right)\end{array}$ & $\begin{array}{c}\text { MIN } \\
\text { WIND } \\
(\mathrm{KM} / \mathrm{HR})\end{array}$ & $\begin{array}{c}\text { MAX } \\
\text { WIND } \\
(\mathrm{KM} / \mathrm{HR})\end{array}$ & $\begin{array}{l}\text { MIN } \\
\text { SMOW } \\
\text { (CM) }\end{array}$ & $\begin{array}{l}\text { MAX } \\
\text { SNOW } \\
\text { (CM) }\end{array}$ & SKY A.M. & SKY P.M. \\
\hline Archerwill & 27 Dec 2019 & -13 & -18 & 12 & 18 & 8 & 8 & clear & clear \\
\hline Avonlea & 27 Dec 2019 & -2 & -2 & 0 & 30 & 0 & 10 & clear & clear \\
\hline Balgonie & 4 Jan 2020 & -6 & 3 & 15 & 28 & 0 & 5 & cloudy & partly cloudy \\
\hline Biggar & 18 Dec 2019 & -5 & 1 & 14 & 16 & 0 & 10 & partly cloudy & partly cloudy \\
\hline Birch Hills & 31 Dec 2019 & -15 & -13 & 4 & 11 & 4 & 10 & heavy fog & heavy fog \\
\hline Borden-Radisson & 21 Dec 2019 & -10 & -7 & 0 & 15 & & & cloudy & partly cloudy \\
\hline Broadview & 15 Dec 2019 & -12 & -10 & 10 & 15 & 4 & 6 & overcast & light snow \\
\hline Candle Lake & 4 Jan 2020 & -9 & -5 & 15 & 35 & 10 & 15 & overcast & overcast \\
\hline Cater & 3 Jan 2020 & -10 & -7 & 0 & 10 & 10 & 18 & overcast & overcast \\
\hline Chatsworth S.D. & 4 Jan 2020 & -10 & -6 & 25 & 35 & 10 & 25 & cloudy & cloudy \\
\hline Churchbridge A & & & & & & & & & overcast \\
\hline Churchbridge B & 27 Dec 2019 & -12 & -8 & 20 & 29 & 15 & 20 & & partly cloudy \\
\hline Clark's Crossing & 14 Dec 2019 & -27 & -18 & 5 & 11 & 3 & 10 & mostly clear & mostly clear \\
\hline Craven & 14 Dec 2019 & -26 & -19 & 11 & 20 & 0 & 5 & partly cloudy & cloudy \\
\hline Crooked Lake & 18 Dec 2019 & -10 & -1 & 10 & 20 & 6 & 15 & mostly clear & mostly clear \\
\hline Crooked River & 30 Dec 2019 & -13 & -12 & 2 & & 15 & 15 & clear & mostly clear \\
\hline Cypress Hills P.P. & 28 Dec 2019 & -10 & 2 & 2 & 2 & 8 & 20 & clear & clear \\
\hline Denholm & 25 Dec 2019 & -5 & -2 & 0 & 8 & 5 & 10 & overcast & overcast \\
\hline Dorintosh & 29 Dec 2019 & -11 & -5 & 2 & 2.5 & 10 & 12 & cloudy & partly cloudy \\
\hline Duval & 18 Dec 2019 & -12 & -5 & 0 & 10 & 0 & 32 & partly cloudy & cloudy \\
\hline E.B.Campbell Dam & 30 Dec 2019 & -11 & -6 & 5 & 10 & 10 & 40 & light snow & overcast \\
\hline Eastend & 4 Jan 2020 & 0 & 1 & 20 & 50 & 0 & 10 & overcast & overcast \\
\hline Ebenezer B & 26 Dec 2019 & -10 & -5 & 2 & 5 & 12 & 12 & mostly clear & mostly clear \\
\hline Estevan & 2 Jan 2020 & -2 & -1 & 8 & 16 & 0 & 10 & overcast & overcast \\
\hline Estuary North & 5 Jan 2020 & -3 & 3 & 0 & 8 & 2 & 4 & clear & clear \\
\hline Fenton & 25 Dec 2019 & -6 & -5 & 6 & 10 & 10 & 10 & mod. Snow & mod. snow \\
\hline Floral & 29 Dec 2019 & -18 & -10 & 8 & 14 & 5 & 10 & partly cloudy & overcast \\
\hline Fort Qu'Appelle & 17 Dec 2019 & -16 & -12 & 2 & 2 & 5 & 10 & overcast & overcast \\
\hline Gardiner Dam & 16 Dec 2019 & -7 & -5 & 16 & 18 & 0 & 10 & partly cloudy & mostly clear \\
\hline Good Spirit Lake & 3 Jan 2020 & -8 & -3 & 15 & 15 & 10 & 20 & cloudy & cloudy \\
\hline Grayson & 29 Dec 2019 & -16 & -13 & 0 & 10 & 0 & 15 & heavy fog & mostly clear \\
\hline Grenfell & 4 Jan 2020 & -7 & 0 & 18 & 20 & 4 & 6 & cloudy & partly cloudy \\
\hline Harris & 19 Dec 2019 & -10 & -4 & 25 & 35 & 0 & 5 & overcast & partly cloudy \\
\hline Hazlet & 24 Dec 2019 & -6 & -2 & 13 & 22 & 0 & 3 & overcast & cloudy \\
\hline Indian Head & 27 Dec 2019 & -9 & -4 & 10 & 15 & 0 & 12 & clear & partly cloudy \\
\hline Kenaston & 17 Dec 2019 & 0 & 8 & & 10 & & 3 & & \\
\hline Kenosee Lake & 24 Dec 2019 & -6 & -5 & 0 & 10 & 5 & 20 & mod. Fog & light fog \\
\hline
\end{tabular}




\begin{tabular}{|c|c|c|c|c|c|c|c|c|c|}
\hline LOCALITY & DATE & $\begin{array}{c}\text { MIN } \\
\text { TEMP } \\
\left({ }^{\circ} \mathrm{C}\right)\end{array}$ & $\begin{array}{c}\text { MAX } \\
\text { TEMP } \\
\left({ }^{\circ} \mathrm{C}\right)\end{array}$ & $\begin{array}{c}\text { MIN } \\
\text { WIND } \\
\text { (KM/HR) }\end{array}$ & $\begin{array}{c}\text { MAX } \\
\text { WIND } \\
\text { (KM/HR) }\end{array}$ & $\begin{array}{l}\text { MIN } \\
\text { SMOW } \\
\text { (CM) }\end{array}$ & $\begin{array}{l}\text { MAX } \\
\text { SNOW } \\
\text { (CM) }\end{array}$ & SKY A.M. & SKY P.M. \\
\hline Ketchen North & 26 Dec 2019 & -12 & -8 & 10 & 20 & 10 & 12 & clear & clear \\
\hline Kinloch & 29 Dec 2019 & -19 & -11 & 2 & 5 & 18 & 20 & clear & clear \\
\hline Kyle & $21 \operatorname{Dec} 2019$ & -4 & 5 & 2 & 10 & 0 & 5 & overcast & overcast \\
\hline La Ronge & $26 \operatorname{Dec} 2019$ & -13 & -8 & 5 & 20 & 15 & 20 & clear & clear \\
\hline Love-Torch River & 26 Dec 2019 & -25 & -12 & 0 & 5 & & & overcast & overcast \\
\hline Luseland & 26 Dec 2019 & -14 & -8 & 0 & 5 & 2 & 4 & partly cloudy & mostly clear \\
\hline Melfort & 28 Dec 2019 & -15 & -7 & 10 & 20 & & & partly cloudy & partly cloudy \\
\hline Moose Jaw & 21 Dec 2019 & -7 & 7 & 0 & 21 & 0 & 4 & overcast & mostly clear \\
\hline Moose Mountain & 5 Jan 2020 & -9 & -4 & 10 & 26 & 10 & 15 & clear & clear \\
\hline Morse & 23 Dec 2019 & -5 & -1 & 19 & 29 & 10 & 25 & overcast & cloudy \\
\hline Nicolle Flats & 18 Dec 2019 & -4 & 1 & 5 & 25 & 0 & 6 & partly cloudy & partly cloudy \\
\hline Nipawin & 28 Dec 2019 & -12 & -15 & 0 & 2 & 10 & 14 & clear & clear \\
\hline Nisbet Forest West & 28 Dec 2019 & -12 & -7 & 4 & 10 & 5 & 12 & heavy fog & heavy fog \\
\hline Odessa & $24 \operatorname{Dec} 2019$ & -6 & -4 & 5 & 11 & 6 & 12 & light fog & light fog \\
\hline Outlook & 29 Dec 2019 & -13 & -7 & 2 & 10 & 0 & 5 & clear & clear \\
\hline Pike Lake & 4 Jan 2020 & -7 & 2 & 12 & 22 & 3 & 10 & partly cloudy & mostly clear \\
\hline Ponteix & 23 Dec 2019 & -5 & -2 & 10 & 35 & 0 & 15 & light fog & partly cloudy \\
\hline Prince Albert & 22 Dec 2019 & -8 & -6 & 5 & 5 & 10 & 10 & overcast & overcast \\
\hline Qu'Appelle & 15 Dec 2019 & -16 & -11 & 8 & 21 & 5 & 20 & partly cloudy & partly cloudy \\
\hline Qu'Appelle Dam & 16 Dec 2019 & -11 & -6 & 0 & 15 & 0 & 10 & cloudy & partly cloudy \\
\hline Regina & $28 \operatorname{Dec} 2019$ & -17 & -4 & 4 & 4 & 2 & 10 & mostly clear & clear \\
\hline Roscommon S.D. & 1 Jan 2020 & -17 & -14 & 11 & 29 & 0 & 0 & light snow & mostly clear \\
\hline Rouleau & 13 Dec 2019 & -5 & 2 & 20 & 29 & 1 & 2 & partly cloudy & cloudy \\
\hline Round Lake (Q.V.) & 16 Dec 2019 & -6 & -4 & 30 & 39 & 10 & 25 & partly cloudy & cloudy \\
\hline Saltcoats & 21 Dec 2019 & -12 & -3 & 2 & 5 & 8 & 8 & heavy fog & heavy fog \\
\hline Sask. Landing P.P. & 20 Dec 2019 & 2 & 5 & 5 & 20 & 0 & 5 & partly cloudy & clear \\
\hline Sask. River Forks & 21 Dec 2019 & -12 & -10 & 0 & 5 & 3 & 5 & heavy fog & cloudy \\
\hline Saskatoon & 26 Dec 2019 & -16 & -10 & 16 & 23 & 4 & 15 & clear & clear \\
\hline Shamrock & 30 Dec 2019 & -12 & -8 & 12 & 12 & 10 & 16 & clear & clear \\
\hline Shell Lake & 14 Dec 2019 & -33 & -17 & 5 & 10 & 2 & 8 & clear & clear \\
\hline Snowden & 4 Jan 2020 & -3 & -7 & 2 & 5 & 2 & 13 & cloudy & cloudy \\
\hline Swift Current & 14 Dec 2019 & -17 & -11 & 10 & 15 & 15 & 30 & cloudy & cloudy \\
\hline Turtleford & $22 \operatorname{Dec} 2019$ & -8 & -5 & 2 & 6 & 3 & 10 & cloudy & cloudy \\
\hline Weyburn & 28 Dec 2019 & -15 & -8 & 8 & 10 & 10 & 15 & clear & mostly clear \\
\hline White Bear & 3 Jan 2020 & -13 & -7 & 5 & 0 & 0 & 10 & clear & clear \\
\hline Whitewood & 29 Dec 2019 & -14 & -9 & 0 & 10 & 10 & 20 & clear & clear \\
\hline Wingard & $2 \operatorname{Jan} 2020$ & & & & & & & & \\
\hline
\end{tabular}


TABLE 2. Effort and Habitat Coverage (\%). Wild Fruit: $p=$ poor, $f=f a i r, g=g o o d, e=e x c e l l e n t$.

\begin{tabular}{|c|c|c|c|c|c|c|c|c|c|c|c|c|c|c|c|c|c|c|c|c|}
\hline LOCALITY & 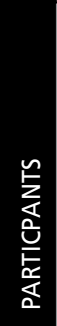 & 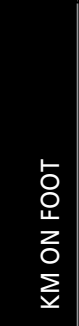 & 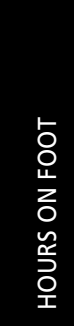 & 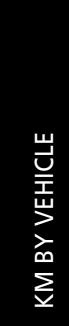 & 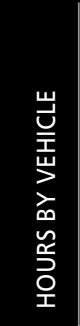 & 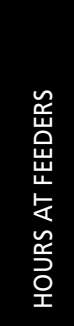 & 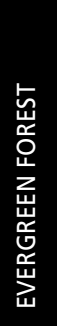 & 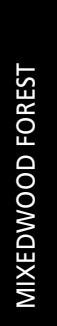 & 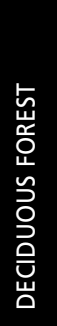 & 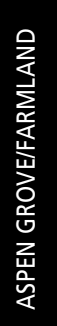 & 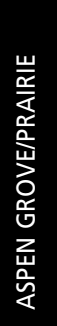 & 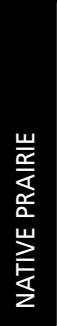 & 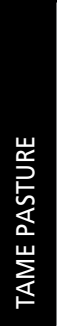 & 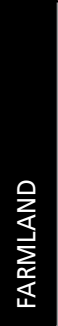 & 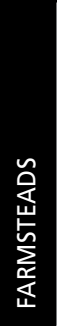 & \begin{tabular}{l}
$z$ \\
$\frac{\alpha}{\alpha}$ \\
ơ \\
\multirow{2}{*}{}
\end{tabular} & 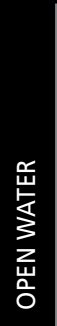 & $\begin{array}{l}\frac{z}{\alpha} \\
\frac{\alpha}{\underline{\alpha}} \\
\frac{\alpha}{\alpha}\end{array}$ & 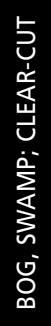 & 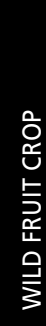 \\
\hline Archerwill & 9 & 3.5 & 0.5 & 0 & 0.0 & 7.5 & & 25 & & & & & & 50 & 25 & & & & & $\mathrm{p}$ \\
\hline Avonlea & 4 & 0.0 & 0.0 & 130 & 7.0 & 1.0 & & & & & & 15 & 10 & 25 & 10 & 35 & & 5 & & \\
\hline Balgonie & 11 & 17.0 & 7.0 & 293 & 9.5 & 0.0 & & & & 20 & & & & 45 & 10 & 25 & & & & $f$ \\
\hline Biggar & 10 & 11.0 & 7.5 & 400 & 9.8 & 7.0 & & & & 23 & & & & 36 & 6 & 35 & & & & $\mathrm{p}$ \\
\hline Birch Hills & 2 & 0.5 & 0.5 & 51 & 5.5 & 1.0 & & & 5 & 30 & 5 & & 5 & 40 & 5 & & & 10 & & g \\
\hline Borden-Radisson & 8 & 15.0 & 8.0 & 255 & 5.3 & 0.0 & & & & & & & & & & & & & & \\
\hline Broadview & 2 & 1.5 & 1.0 & 136 & 5.5 & 2.0 & & & 20 & 25 & & 5 & 10 & 25 & 5 & 10 & & & & $p$ \\
\hline Candle Lake & 6 & 1.0 & 0.5 & 156 & 12.0 & 0.3 & 40 & 30 & & & & & & & 25 & & & & 5 & $\mathrm{p}$ \\
\hline Cater & 2 & 0.0 & 0.0 & 212 & 6.5 & 2.0 & 5 & 10 & 5 & 15 & & & 5 & 50 & 5 & 5 & & & & \\
\hline Chatsworth S.D. & 12 & 4.0 & 7.0 & 108 & 8.0 & 4.0 & & & & 80 & & 10 & 5 & & 5 & & & & & g \\
\hline Churchbridge $A$ & 2 & & & & & & & & & & & & & & & & & & & \\
\hline Churchbridge B & 1 & & & & & & & & & & & & & & & & & & & \\
\hline Clark's Crossing & 31 & 23.8 & 11.8 & 654 & 34.2 & 18.5 & & & & 20 & 5 & 5 & 5 & 20 & 10 & 25 & & 10 & & $\mathrm{p}$ \\
\hline Craven & 34 & 43.1 & 11.8 & 717 & 24.5 & 8.5 & & & & 25 & 15 & 5 & 5 & 20 & 10 & 20 & & & & $f$ \\
\hline Crooked Lake & 1 & 1.0 & 0.5 & 143 & 4.0 & 0.0 & & & & 10 & & & & 40 & 10 & 20 & 20 & & & g \\
\hline Crooked River & 3 & 0.5 & 0.5 & 25 & 1.0 & 5.0 & & 40 & & & & & & 40 & 20 & & & & & $f$ \\
\hline Cypress Hills P.P. & 16 & 8.0 & 5.0 & 20 & 1.0 & 0.0 & 40 & 40 & 8 & & & 10 & & & 2 & & & & & $\mathrm{~g}$ \\
\hline Denholm & 4 & 0.0 & 0.0 & 199 & 7.8 & 0.8 & & & & 10 & 5 & & 5 & 70 & 5 & 5 & & & & $p$ \\
\hline Dorintosh & 4 & 24.8 & 10.0 & 36 & 2.0 & 1.0 & & 60 & & & & & & & 40 & & & & & $f$ \\
\hline Duval & 2 & 2.0 & 1.0 & 126 & 5.8 & 0.3 & & & & 15 & 10 & & & 40 & 10 & 25 & & & & e \\
\hline E.B.Campbell Dam & 8 & 7.0 & 4.5 & 164 & 8.8 & 3.0 & 10 & 45 & 15 & 5 & & & & & & & 20 & 5 & & $f$ \\
\hline Eastend & 12 & & & & & & & & & & & & & & & & & & & $\mathrm{p}$ \\
\hline Ebenezer B & 3 & 3.0 & 0.5 & 5 & 2.5 & 2.0 & & & & & 35 & & 35 & & 5 & & & 25 & & e \\
\hline Estevan & 9 & 8.0 & 3.5 & 252 & 9.5 & 5.0 & & & & & & & & 38 & 8 & 13 & 18 & 23 & & $\mathrm{p}$ \\
\hline Estuary North & 3 & 2.0 & 4.0 & 52 & 10.0 & 2.0 & & & 30 & & & 40 & & 20 & 10 & & & & & $f$ \\
\hline Fenton & 1 & 0.0 & 0.0 & 88 & 3.3 & 0.0 & & & & & & & & & & & & & & \\
\hline Floral & 1 & 5.0 & 2.8 & 191 & 6.0 & 0.0 & & & & 26 & & & & 48 & 12 & 14 & & & & $\mathrm{p}$ \\
\hline Fort Qu'Appelle & 16 & 0.0 & 0.0 & 274 & 16.0 & 0.0 & & & & 25 & 20 & & 10 & 20 & 5 & 20 & & & & $p$ \\
\hline Gardiner Dam & 9 & 20.0 & 13.5 & 450 & 13.8 & 0.0 & & & & 6 & & & & 46 & 11 & 4 & 28 & 5 & & $\mathrm{p}$ \\
\hline Good Spirit Lake & 3 & 0.0 & 0.0 & 117 & 6.0 & 0.0 & & & & 20 & & & 20 & 30 & 5 & 25 & & & & $f$ \\
\hline Grayson & 7 & 4.0 & 2.0 & 160 & 7.0 & 5.0 & & & & 60 & & & & 5 & 10 & 10 & 5 & 10 & & $f$ \\
\hline Grenfell & 3 & 0.0 & 0.0 & 129 & 7.0 & 2.0 & & & & 5 & 20 & 15 & 5 & 15 & 25 & & 15 & & & $p$ \\
\hline Harris & 5 & 8.0 & 6.3 & 183 & 6.5 & 0.0 & & & & 14 & & & & 33 & 25 & 28 & & & & $f$ \\
\hline Hazlet & 1 & 1.8 & 0.5 & 257 & 6.1 & 0.0 & & & & & & 10 & 15 & 65 & 5 & 5 & & & & $f$ \\
\hline Indian Head & 24 & 10.5 & 3.0 & 667 & 6.0 & 30.0 & 5 & & & 10 & & & & 75 & 5 & 5 & & & & $\mathrm{p}$ \\
\hline Kenaston & 3 & 0.0 & 0.0 & 123 & 2.0 & 2.0 & & & & & & & & 90 & 5 & 5 & & & & \\
\hline Kenosee Lake & 2 & 0.1 & 2.0 & 154 & 3.5 & 0.0 & & & 20 & 10 & 10 & 5 & 5 & & & 50 & & & & $f$ \\
\hline
\end{tabular}




\begin{tabular}{|c|c|c|c|c|c|c|c|c|c|c|c|c|c|c|c|c|c|c|c|c|}
\hline LOCALITY & 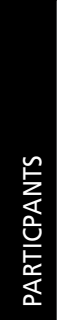 & $\begin{array}{l}\llcorner \\
\text { o } \\
\mathbf{4} \\
\mathbf{z} \\
\sum_{\underline{z}}\end{array}$ & 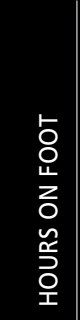 & 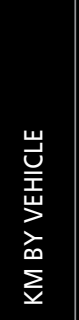 & 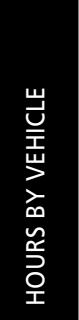 & 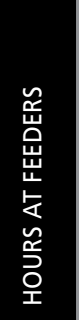 & 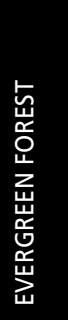 & 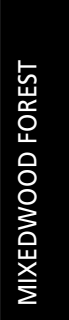 & 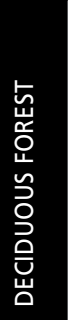 & 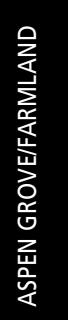 & 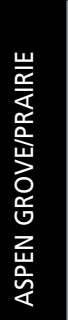 & 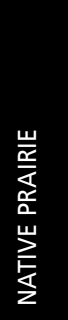 & 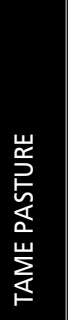 & 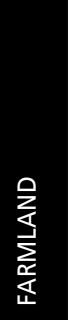 & 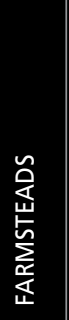 & $\begin{array}{l}z \\
\frac{\alpha}{\infty} \\
\text { 产 }\end{array}$ & 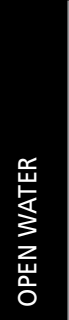 & $\begin{array}{l}\frac{z}{\alpha} \\
\frac{\alpha}{\frac{\alpha}{\alpha}} \\
\frac{0}{\alpha}\end{array}$ & 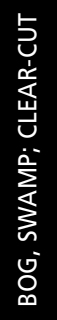 & 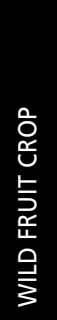 \\
\hline Ketchen North & 1 & 1.5 & 1.0 & 60 & 3.0 & 5.5 & & & & 30 & & & 20 & 20 & 20 & 10 & & & & \\
\hline Kinloch & 5 & 2.0 & 1.0 & 86 & 7.0 & 10.0 & 5 & 30 & 20 & 30 & & & & & 15 & & & & & $\mathrm{~g}$ \\
\hline Kyle & 4 & 10.0 & 4.5 & 142 & 19.5 & 0.0 & & & & & 15 & & & 50 & 25 & & & 10 & & $\mathrm{~g}$ \\
\hline La Ronge & 5 & 3.0 & 3.0 & 48 & 3.0 & 4.0 & & 10 & & & & & & & & 80 & & 10 & & $\mathrm{~g}$ \\
\hline Love-Torch River & 16 & 6.0 & 3.5 & 405 & 14.5 & 13.0 & 10 & 15 & 5 & 50 & & & & & 10 & 10 & & & & $\mathrm{p}$ \\
\hline Luseland & 7 & 7.0 & 2.5 & 311 & 7.5 & 2.0 & & & & 50 & & 5 & 5 & 25 & 5 & 10 & & & & $f$ \\
\hline Melfort & 8 & 0.0 & 0.0 & 94 & 5.0 & 6.8 & & & & 5 & & & & 45 & & 50 & & & & $f$ \\
\hline Moose Jaw & 6 & 23.1 & 11.7 & 298 & 11.1 & 0.0 & & 10 & & & & & 5 & 40 & 5 & 30 & & 10 & & $f$ \\
\hline Moose Mountain & 4 & 0.0 & 0.0 & 180 & 5.5 & 2.0 & & 54 & & & & 2 & & 31 & 3 & 10 & & & & $\mathrm{~g}$ \\
\hline Morse & 10 & 5.5 & 1.5 & 466 & 23.3 & 3.0 & & & & & & & 3 & 90 & 7 & & & & & f \\
\hline Nicolle Flats & 5 & 8.5 & 3.2 & 283 & 11.8 & 0.5 & & & 25 & & & 17 & 5 & 40 & 1 & 9 & 1 & 2 & & $f$ \\
\hline Nipawin & 16 & 1.0 & 0.5 & 226 & 7.0 & 7.0 & 15 & 5 & & 15 & & & & 25 & 10 & 30 & & & & $f$ \\
\hline Nisbet Forest West & 3 & 1.0 & 0.5 & 100 & 4.5 & 0.0 & 10 & 10 & 20 & 30 & & & & 30 & & & & & & $f$ \\
\hline Odessa & 3 & 0.0 & 0.0 & 60 & 4.0 & 8.0 & & & & 25 & & 25 & 25 & 10 & 15 & & & & & $f$ \\
\hline Outlook & 1 & 0.0 & 0.0 & 30 & 4.0 & 0.0 & & & & 70 & 10 & & & & 7 & 10 & 1 & 2 & & \\
\hline Pike Lake & 32 & 30.1 & 26.3 & 389 & 14.6 & 8.5 & & & 5 & 5 & 5 & & 5 & 40 & 20 & 15 & & 5 & & $\mathrm{p}$ \\
\hline Ponteix & 1 & 3.7 & 0.8 & 276 & 7.0 & 0.0 & & & & & & 5 & 20 & 55 & 5 & 15 & & & & $\mathrm{p}$ \\
\hline Prince Albert & 26 & 34.0 & 18.0 & 474 & 27.6 & 1.5 & & & & & & & & & & & & & & $\mathrm{~g}$ \\
\hline Qu'Appelle & 7 & 1.5 & 0.8 & 321 & 11.5 & 5.0 & & 2 & & 30 & 5 & & 8 & 10 & 10 & 25 & 5 & 5 & & $\mathrm{f}$ \\
\hline Qu'Appelle Dam & 10 & 3.5 & 2.8 & 527 & 23.0 & 4.0 & & & & 30 & 10 & & & 20 & 10 & 15 & 5 & 10 & & $f$ \\
\hline Regina & 49 & 95.5 & 35.9 & 645 & 19.9 & 0.0 & & & & 10 & & & & 25 & 5 & 60 & & & & $f$ \\
\hline Roscommon S.D. & 10 & 2.0 & 1.0 & 88 & 2.5 & 24.0 & & & & 20 & & & & 60 & 20 & & & & & e \\
\hline Rouleau & 4 & 0.5 & 0.5 & 369 & 7.5 & 1.0 & & & & 5 & & & & 80 & 5 & 10 & & & & $\mathrm{p}$ \\
\hline Round Lake (Q.V.) & 4 & 0.0 & 0.0 & 92 & 5.0 & 8.0 & & & & & & & 10 & 40 & 10 & 25 & 15 & & & $g$ \\
\hline Saltcoats & 23 & 0.0 & 0.0 & 60 & 8.0 & 22.5 & & & & & 2 & 10 & 5 & 80 & & & 3 & & & \\
\hline Sask. Landing P.P. & 5 & 15.0 & 6.5 & 190 & 25.0 & 0.0 & & & & 5 & & & 10 & & 45 & 10 & & 30 & & $f$ \\
\hline Sask. River Forks & 2 & 2.0 & 1.0 & 83 & 5.0 & 0.0 & 15 & 20 & 20 & 15 & & & 5 & 20 & 5 & & & & & $\mathrm{p}$ \\
\hline Saskatoon & 77 & 107.3 & 46.4 & 848 & 47.1 & 48.9 & & & & 10 & & & & 5 & 5 & 75 & & 5 & & $f$ \\
\hline Shamrock & 8 & 3.5 & 2.5 & 390 & 16.5 & 1.0 & & & & & & & 5 & 60 & 25 & 10 & & & & \\
\hline Shell Lake & 8 & 7.3 & 3 & 265 & 8.4 & 0.5 & 5 & $<1$ & 25 & 5 & 5 & 5 & 30 & 15 & $<1$ & 5 & & 5 & & $p$ \\
\hline Snowden & 9 & & & & 4.5 & 2.0 & & & & & & & & & 90 & 10 & & & & $\mathrm{e}$ \\
\hline Swift Current & 28 & 32.5 & 14.8 & 368 & 22.8 & 10.3 & & & & & & & & 20 & 5 & 70 & & 5 & & $\mathrm{~g}$ \\
\hline Turtleford & 14 & 12.0 & 4.0 & 380 & 8.0 & 13.0 & & & & 45 & & & & 50 & & 5 & & & & $\mathrm{~g}$ \\
\hline Weyburn & 17 & 0.0 & 0.0 & 320 & 12.0 & 4.0 & & & 2 & & 2 & 5 & 8 & 58 & 10 & 15 & & & & $\mathrm{~g}$ \\
\hline White Bear & 5 & 15.0 & 6.0 & 310 & 21.5 & 0.0 & & & & & & 5 & 5 & 60 & 25 & & & 5 & & $\mathrm{f}$ \\
\hline Whitewood & 18 & -14.0 & -9.0 & 271 & 13.5 & 21.0 & & & & 10 & & & & 15 & 5 & 70 & & & & $\mathrm{~g}$ \\
\hline Wingard & 1 & & & & & & & & & & & & & & & & & & & \\
\hline
\end{tabular}


TABLE 3. Species found on 5 or more counts; ()$=$ seen during count period (CP); CP totals include CP birds listed in Table 4.

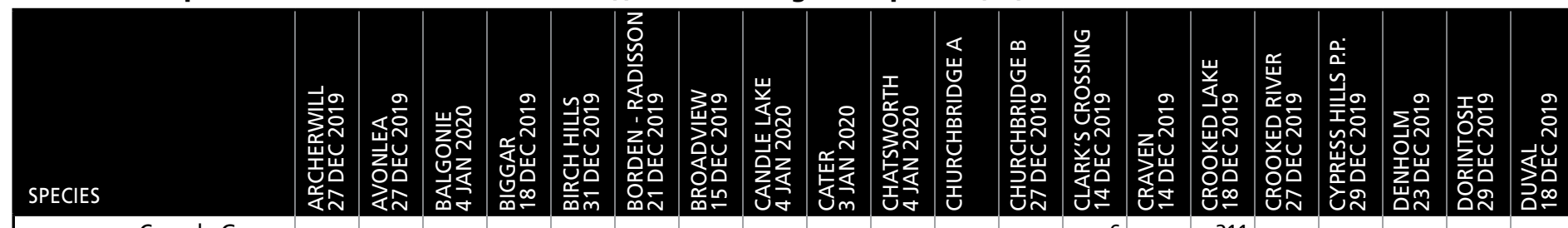

Canada Goose Mallard

Common Goldeneye

Ring-necked Pheasant Gray Partridge Ruffed Grouse

Sharp-tailed Grouse Rock Pigeon

Eurasian Collared-Dove Golden Eagle

Sharp-shinned Hawk

Northern Goshawk Bald Eagle

Great Horned Owl Snowy Owl

Northern Saw-whet Owl

American Three-toed Woodpecker Black-backed Woodpecker

Downy Woodpecker Hairy Woodpecker

Pileated Woodpecker Northern Flicker Merlin Gyrfalcon Prairie Falcon

Northern Shrike Canada Jay Blue Jay

Black-billed Magpie Common Raven Black-capped Chickadee Boreal Chickadee Horned Lark

Golden-crowned Kinglet Red-breasted Nuthatch White-breasted Nuthatch Brown Creeper European Starling American Robin Bohemian Waxwing Cedar Waxwing House Sparrow Evening Grosbeak Pine Grosbeak Gray-crowned Rosy-Finch House Finch Purple Finch Common Redpoll Red Crossbill Pine Siskin

American Goldfinch Snow Bunting

Dark-eyed Junco

White-throated Sparrow Rusty Blackbird TOTAL BIRDS COUNT DAY TOTAL BIRDS ONLY IN CP TOTAL SPECIES COUNT DAY TOTAL SPECIES ONLY IN CP
(8)

(1)

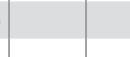

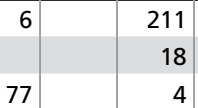

211
18

4

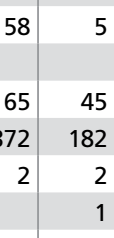

(1)

\section{(1)}

(1)

(1)

\section{4}

1

1

(5) 15 \begin{tabular}{l|l}
28 & 5
\end{tabular}

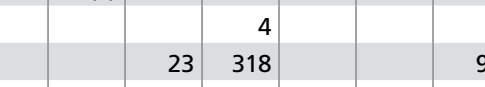

\section{0}

114

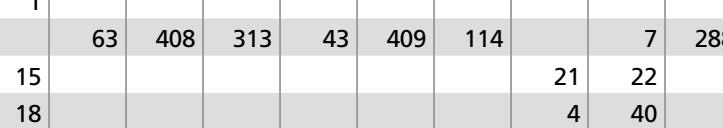

18
2

98
85

(1)

\section{\begin{tabular}{|l|l|l|}
\hline $57 \quad(10)$ & 16 & 30
\end{tabular}} \begin{tabular}{r|r}
108 & 24 \\
2 & 3
\end{tabular} \begin{tabular}{r|r|}
2 & 3 \\
36 & 290
\end{tabular}
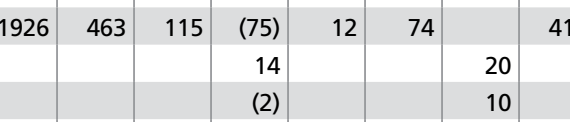

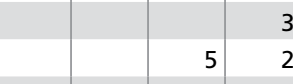

3
2

\begin{tabular}{|r|r|r|r|r|r|}
\hline 40 & & 40 & 761 & 69 & 12 \\
\hline$(3)$ & 2 & 1 & 1 & 1 & \\
\hline
\end{tabular}

(3)

\begin{tabular}{r|r|r|r|r|r|r|r|r|r|r|}
142 & 224 & 1124 & 1875 & 129 & 978 & 289 & 433 & 403 & 541 & 46 \\
$(13)$ & $(5)$ & $(1)$ & 0 & 0 & 0 & $(10)$ & 0 & 0 & 0 & 0 \\
14 & 17 & 26 & 23 & 10 & 25 & 15 & 16 & 14 & 14 & 6 \\
4 & 1 & 1 & 0 & 0 & 0 & 6 & 0 & 0 & 0 & 0
\end{tabular}

\begin{tabular}{|c|c|c|c|c|c|c|c|c|}
\hline 43 & 3815 & 1588 & 460 & 42 & 143 & 1130 & 77 & 574 \\
\hline 0 & (1) & (1) & 0 & (87) & (1) & (8) & (1) & (9) \\
\hline 8 & 26 & 29 & 12 & 10 & 12 & 12 & 12 & 14 \\
\hline 0 & 1 & 1 & 0 & 3 & 1 & 4 & 1 & \\
\hline
\end{tabular}




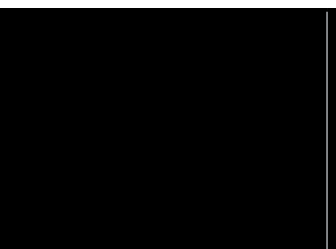

Canada Goose Mallard

Common Goldeneye Ring-necked Pheasant Gray Partridge Ruffed Grouse Sharp-tailed Grouse Rock Pigeon Eurasian Collared-Dove Golden Eagle Sharp-shinned Hawk Northern Goshawk Bald Eagle

Great Horned Owl Snowy Ow

Northern Saw-whet Owl

American Three-toed Woodpecker

Black-backed Woodpecker

Downy Woodpecker Hairy Woodpecker Pileated Woodpecker

Northern Flicker Merlin Gyrfalcon Prairie Falcon Northern Shrike Canada Jay Blue Jay

Black-billed Magpie Common Raven

Black-capped Chickadee Boreal Chickadee Horned Lark Golden-crowned Kinglet Red-breasted Nuthatch White-breasted Nuthatch Brown Creeper European Starling American Robin

Bohemian Waxwing Cedar Waxwing House Sparrow Evening Grosbeak Pine Grosbeak

Gray-crowned Rosy-Finch House Finch Purple Finch Common Redpoll Red Crossbill Pine Siskin

American Goldfinch Snow Bunting Dark-eyed Junco White-throated Sparrow Rusty Blackbird

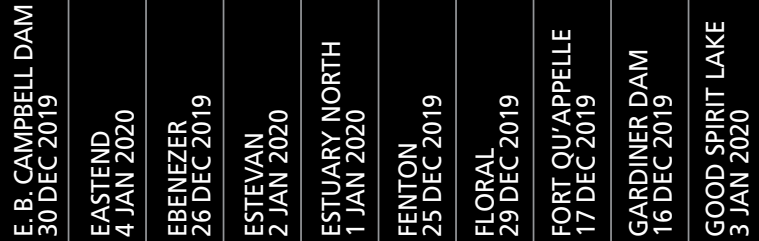
33775 5631

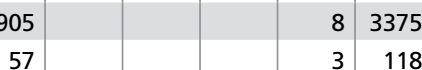
\begin{tabular}{|l|r|}
\hline 1076 & 57 \\
\hline
\end{tabular}

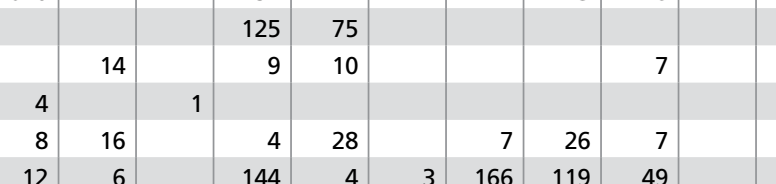
\begin{tabular}{|l|l|r|r|r|r|r|r|r|r|}
\hline & 33 & & 16 & 8 & & & & 12 \\
\hline$(10)$ & & & & 4 & & $(1)$ & 7 & 2 & \\
\hline$(12)$ & 13 & 12 & 40 & 34 & 15 & 5 & & & 32 \\
\hline
\end{tabular} \begin{tabular}{|l|r|r|r|r|r|r} 
(12) & 13 & 12 & 40 & 34 & 15 & 5 \\
\hline
\end{tabular} \begin{tabular}{|r|r|r|r|r|r|r|r|}
\hline 12 & 144 & 4 & 3 & 166 & 119 & 49 \\
\hline & 40 & 14 & 2 & & 1 & 18 \\
\hline 3 & & & 1 & & & \\
\hline
\end{tabular}

\begin{tabular}{|l|l|l|l|}
\hline 8 & 3 & 1 & 4 \\
\hline & 1 & 3 & 1 \\
\hline & 2 & 3 & 5 \\
\hline
\end{tabular}

(1) $\quad 1$ $\quad 22$ \begin{tabular}{|r|r|r|r|r|r|r|}
14 & 114 & 85 & 83 & 146 & 2 & 9 \\
\hline
\end{tabular} 401 20

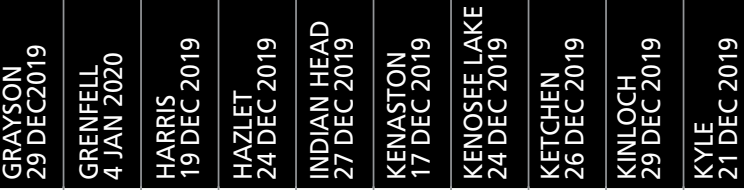
24 2 \begin{tabular}{|l|r|r|r|}
\hline 2 & 1 & 11 & 1 \\
\hline
\end{tabular} (1) $14 \quad 8$ 1

3

\begin{tabular}{|r|r|r|r|r|}
\hline 3 & & & & 1 \\
\hline 1 & 1 & 1 & $(1)$ \\
\hline 4 & $(1)$ & & 9 \\
\hline
\end{tabular}

\begin{tabular}{|c|c|c|c|c|c|c|c|c|c|c|c|}
\hline 1 & 7 & 4 & 8 & 2 & 4 & 17 & 5 & 3 & 4 & 2 & 2 \\
\hline 10 & 2 & 4 & 4 & 2 & 2 & 9 & 1 & & 2 & 1 & 1 \\
\hline 4 & & & & 1 & & & & & & & \\
\hline & 4 & & & 3 & & 1 & 1 & & & & \\
\hline
\end{tabular}

\begin{tabular}{|l|l|l|l|l|r|}
\hline 21 & 1 & 5 & 4 & 1 & 1 \\
\hline 15 & 1 & 4 & 5 & 2 & 2 \\
\hline & & & 2 & & \\
\hline
\end{tabular}
\begin{tabular}{|l|r|r|r|r|r|r|r|r|r|r|r|r|r|r|r|r|r|r|r|r|r|}
\hline 13 & 38 & 14 & 27 & 12 & 1 & 53 & 109 & 17 & 17 & 36 & 12 & 26 & & 120 & 2 & 82 & 9 & 30 & 6 \\
\hline
\end{tabular}

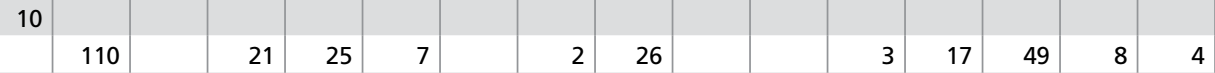

\begin{tabular}{|c|c|c|c|c|c|c|c|c|c|c|c|c|c|c|c|c|c|c|c|}
\hline & & & & & & & & & & & & 1 & & & & & & & \\
\hline \multirow[t]{7}{*}{3} & 6 & & & 3 & & 1 & 5 & 1 & & & 1 & 4 & & 36 & (1) & & & & 1 \\
\hline & 7 & 1 & 10 & 1 & & 2 & 14 & 1 & 1 & 1 & 1 & & & 25 & & 11 & & 3 & \\
\hline & & & & & & & & 1 & & & 1 & & & & & & & & \\
\hline & 3 & & 2 & 250 & & & 10 & 26 & & & 5 & & 8 & 7 & & & & & 26 \\
\hline & & & (1) & & & & 1 & 1 & & & & 1 & & & & & & & \\
\hline & 51 & & 12 & & & 12 & 165 & 102 & & (40) & 45 & 47 & & 14 & & & & (19) & 59 \\
\hline & & 12 & & 4 & & & 8 & & & & & & & & & & & & \\
\hline (1) & 183 & 24 & 162 & 180 & 5 & 324 & 151 & 347 & 7 & 141 & 240 & 419 & 285 & 320 & 75 & 98 & 56 & 6 & 580 \\
\hline 15 & & & & & & & & & & & & & & & & & & 9 & \\
\hline
\end{tabular}

\begin{tabular}{|l|l|l|l|l|l|}
\hline 6 & $(12)$ & 30 & $(2)$ & 6 & 1 \\
\hline
\end{tabular}

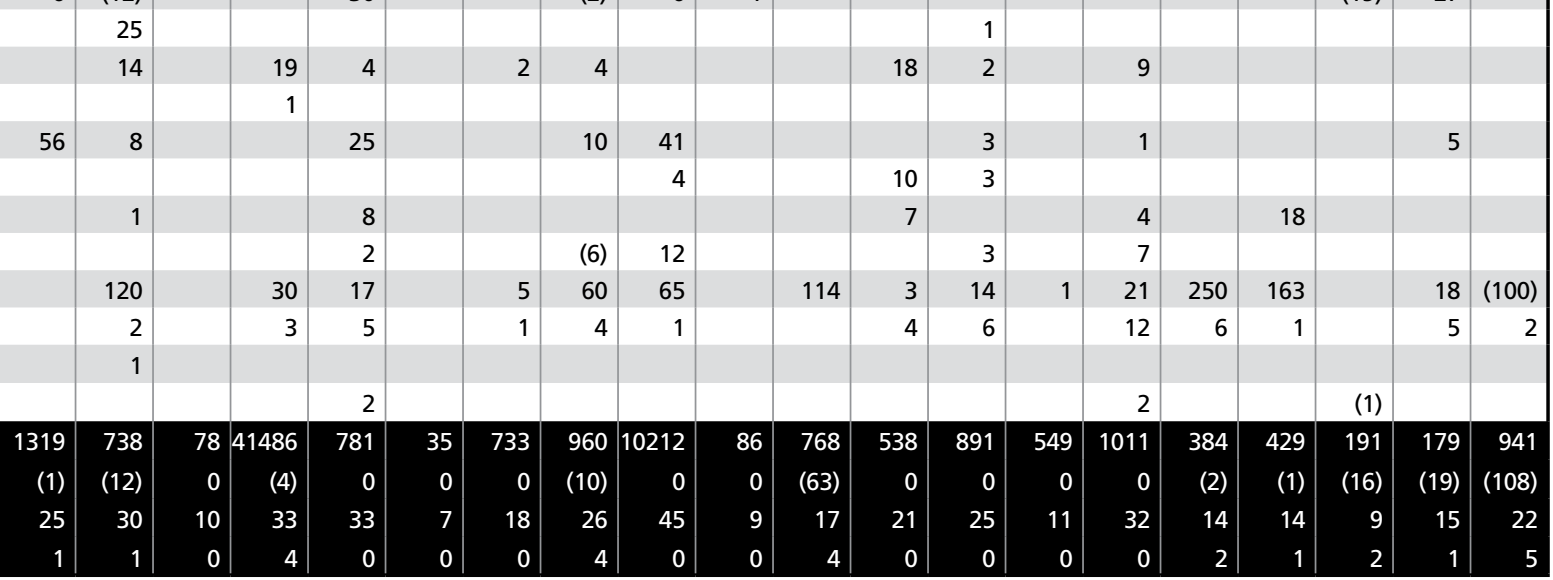


TABLE 3. Species found on 5 or more counts; ()$=$ seen during count period (CP); CP totals include CP birds listed in Table 4.

\begin{tabular}{|c|c|c|c|c|c|c|c|c|c|c|c|c|c|c|c|c|c|c|c|c|}
\hline SPECIES & 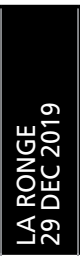 & 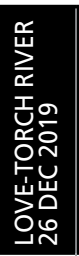 & 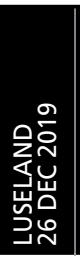 & 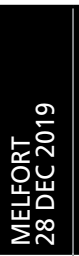 & 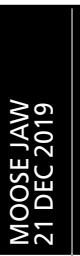 & 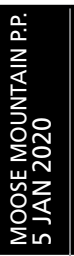 & 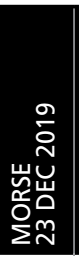 & 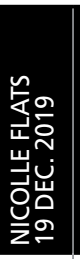 & 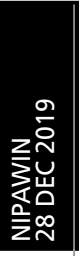 & 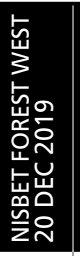 & 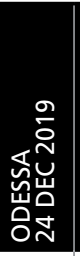 & 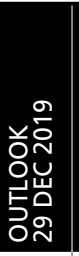 & 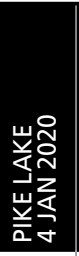 & 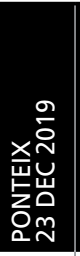 & 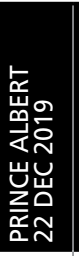 & 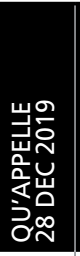 & 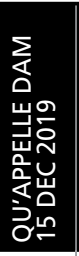 & 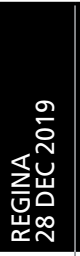 & 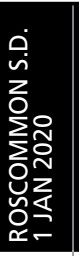 & 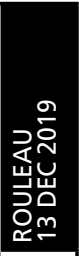 \\
\hline Canada Goose & & & & & 1 & & & & & & & (1) & & & & & 2852 & 295 & & \\
\hline Mallard & & & & & (5) & & & & & & & & & & & & 36 & 120 & & \\
\hline Common Goldeneye & & & & & & & & & & & & & 4 & & & & 6 & & & \\
\hline Ring-necked Pheasant & & & & & 19 & & & & & & & & & (1) & & & & & & \\
\hline Gray Partridge & & 4 & 8 & & 11 & & 321 & 16 & & & 12 & & 20 & 62 & & & & 38 & 5 & \\
\hline Ruffed Grouse & (2) & 4 & & & & 1 & & & 2 & & 6 & & (1) & & 3 & (2) & & & 3 & \\
\hline Sharp-tailed Grouse & 2 & & 3 & & & & 41 & 4 & & (1) & 24 & & 51 & 41 & & 21 & 5 & 14 & & \\
\hline Rock Pigeon & & 103 & 58 & 29 & 493 & 6 & 225 & 32 & 171 & & 12 & 81 & 186 & 171 & 608 & 129 & 36 & 549 & 2 & 117 \\
\hline Eurasian Collared-Dove & & 1 & 3 & 2 & 80 & & 29 & & & & & (1) & & 12 & & 1 & 2 & & & \\
\hline Golden Eagle & & & & & & & & & & & & & & 1 & & & & & & \\
\hline Sharp-shinned Hawk & & & & 1 & & & & 1 & & & & & & & & & & 1 & & \\
\hline Northern Goshawk & & 1 & & & & & & & & & & & & & 3 & & & (1) & (1) & \\
\hline Bald Eagle & & & & & & & & & 3 & & 4 & & 3 & & & (1) & 7 & & (1) & \\
\hline Great Horned Owl & & & 4 & & 2 & & 15 & & & 1 & 4 & 1 & 3 & 2 & 2 & 1 & 4 & 11 & & 4 \\
\hline Snowy Owl & & & 1 & 1 & 7 & & 11 & 1 & & & 2 & & & 15 & & & 1 & 8 & & 2 \\
\hline Northern Saw-whet Owl & & & & & & & 1 & & & & & & 1 & & & & & & & \\
\hline $\begin{array}{r}\text { American Three-toed } \\
\text { Woodpecker }\end{array}$ & & & & & & & & & & & & & & & (1) & & & & & \\
\hline $\begin{array}{l}\text { Black-backed } \\
\text { Woodpecker }\end{array}$ & 1 & (1) & & & & & & & & & & & & & 2 & & & 1 & & \\
\hline Downy Woodpecker & 6 & 12 & 1 & 3 & 1 & & & 1 & 2 & & 4 & 1 & 29 & & 8 & 10 & 7 & 25 & 6 & \\
\hline Hairy Woodpecker & 4 & 13 & (1) & 2 & 1 & 5 & & 1 & 2 & 1 & 3 & & 22 & & 15 & 10 & 5 & 4 & 10 & \\
\hline Pileated Woodpecker & & (1) & & & & & & & & & 2 & & 1 & & 1 & & & 1 & 2 & \\
\hline Northern Flicker & & & & 1 & & & & & 1 & & 2 & & & & & & 2 & 2 & & \\
\hline Merlin & & & (1) & & 2 & & (1) & & & & & & & & & & & 2 & & \\
\hline Gyrfalcon & & & & & & & & & & & & & 1 & & (1) & & 1 & & & \\
\hline Prairie Falcon & & & & & & & & & & & & & & & & & & 1 & & \\
\hline Northern Shrike & & & & & & & 1 & & & & & & 1 & 1 & 2 & & & 1 & & \\
\hline Canada Jay & 5 & 19 & & & & & & & 4 & & & & & & 6 & & & & & \\
\hline Blue Jay & 7 & 34 & 3 & 2 & 1 & 8 & & & 21 & 8 & & 2 & 20 & & 53 & & 1 & 14 & 21 & \\
\hline Black-billed Magpie & 5 & 127 & 111 & 19 & 91 & 22 & 46 & 97 & 22 & 14 & 36 & 24 & 137 & 27 & 222 & 136 & 63 & 98 & 30 & 1 \\
\hline Common Raven & 96 & 227 & 9 & 54 & 12 & 16 & 7 & 51 & 301 & 7 & 22 & 12 & 96 & 1 & 335 & 76 & 89 & 252 & 21 & \\
\hline Black-capped Chickadee & 14 & 101 & 4 & 15 & 36 & 31 & & 13 & 70 & 26 & 20 & (12) & 317 & 1 & 207 & 72 & 36 & 164 & 66 & \\
\hline Boreal Chickadee & 3 & 8 & & & & & & & 1 & 1 & & & & & 3 & & & & & \\
\hline Horned Lark & & & 37 & & 1 & & 144 & 10 & & & & & 4 & 546 & & 1 & 1 & 8 & & 10 \\
\hline Golden-crowned Kinglet & & & & & 3 & & & & & & & & & & & & & 9 & & \\
\hline Red-breasted Nuthatch & 5 & 17 & 2 & 5 & 14 & 5 & 7 & 2 & 7 & & & 2 & 10 & & 13 & 7 & 2 & 113 & & \\
\hline White-breasted Nuthatch & & 7 & 1 & 1 & 11 & 6 & & & 2 & 1 & & (1) & 25 & & 19 & 4 & 1 & 14 & 9 & \\
\hline Brown Creeper & & & & 1 & 1 & & & & & & & & & & 1 & (2) & & 14 & & \\
\hline European Starling & & 13 & & & 109 & & 29 & 4 & 220 & & 14 & & 2 & & & & 45 & 42 & 15 & \\
\hline American Robin & & & & & & & & & & & & & 5 & & (1) & (1) & & (1) & & \\
\hline Bohemian Waxwing & (36) & 106 & 25 & 25 & 15 & & 3 & & 50 & & & & 227 & 12 & 1149 & & 819 & & 31 & \\
\hline Cedar Waxwing & 8 & & & & & & & & & & & & & & & & & & & \\
\hline House Sparrow & & 72 & 112 & 138 & 820 & 4 & 2018 & 45 & 71 & 12 & 36 & 207 & 585 & 875 & 590 & 172 & 339 & 3004 & 50 & 11 \\
\hline Evening Grosbeak & (16) & 316 & & & & & & & 106 & 74 & & & & & 35 & & & & & \\
\hline Pine Grosbeak & 32 & 48 & & & & & & & 34 & 8 & 4 & & 32 & & 5 & 1 & 6 & 3 & & \\
\hline Gray-crowned Rosy-Finch & 1 & & & & & & (1) & & & & & & & & & & & & & \\
\hline House Finch & & & 2 & & 13 & & & & & & & (25) & & & 6 & & 5 & 160 & & \\
\hline Purple Finch & & 1 & & & & 3 & & & & & & & & & (1) & & & 1 & & \\
\hline Common Redpoll & 63 & 56 & & & & & & & 10 & & & & 5 & & 24 & 6 & 50 & & & \\
\hline Red Crossbill & & & & & & & 3 & & & & & & 11 & & 11 & 6 & & 1 & & \\
\hline Pine Siskin & & 8 & & & & & & & 8 & & & & & & 14 & & & & & \\
\hline American Goldfinch & & & & & & & & & & & & & & & 8 & & & & & \\
\hline Snow Bunting & & 436 & 37 & & 20 & & 70 & 67 & & & 40 & & 742 & 6 & 34 & 100 & 180 & 1 & 220 & 261 \\
\hline Dark-eyed Junco & 1 & 1 & & & 1 & 10 & 4 & 4 & 12 & & & & 1 & & 11 & 1 & 1 & 23 & 3 & \\
\hline White-throated Sparrow & & & & & & & & & & & & & (1) & & 2 & & & (1) & & \\
\hline Rusty Blackbird & & & & & & & & & & & 8 & & & & & & & & & \\
\hline TOTAL BIRDS COUNT DAY & 254 & 1736 & 421 & 299 & 1768 & 117 & 2978 & 349 & 1120 & 153 & 261 & 330 & 2543 & 1773 & 3392 & 754 & 4605 & 4997 & 494 & 406 \\
\hline TOTAL BIRDS ONLY IN CP & (54) & (2) & (2) & 0 & (5) & 0 & (2) & 0 & 0 & (1) & 0 & (40) & (2) & (1) & (5) & (7) & 0 & (3) & (2) & 0 \\
\hline TOTAL SPECIES COUNT DAY & 17 & 26 & 18 & 16 & 27 & 12 & 19 & 16 & 22 & 11 & 23 & 8 & 29 & 15 & 30 & 18 & 31 & 36 & 16 & 7 \\
\hline TOTAL SPECIES ONLY IN CP & 3 & 2 & 2 & 0 & 1 & 0 & 2 & 0 & 0 & 1 & 0 & 5 & 2 & 1 & 5 & 5 & 0 & 3 & 2 & 0 \\
\hline
\end{tabular}




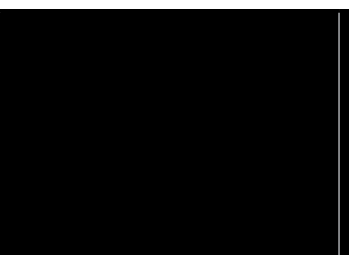

Canada Goose Mallard

Common Goldeneye Ring-necked Pheasant Gray Partridge Ruffed Grouse

Sharp-tailed Grouse Rock Pigeon Eurasian Collared-Dove Golden Eagle Sharp-shinned Hawk Northern Goshawk Bald Eagle

Great Horned Owl Snowy Owl

Northern Saw-whet Owl

American Three-toed Woodpecker

Black-backed

Woodpecker

Downy Woodpecker

Hairy Woodpecker

Pileated Woodpecker

Northern Flicker Merlin

Gyrfalcon

Prairie Falcon

Northern Shrike

Canada Jay Blue Jay

Black-billed Magpie Common Raven Black-capped Chickadee Boreal Chickadee Horned Lark Golden-crowned Kinglet Red-breasted Nuthatch White-breasted Nuthatch Brown Creeper

European Starling American Robin

Bohemian Waxwing Cedar Waxwing House Sparrow Evening Grosbeak Pine Grosbeak

Gray-crowned Rosy-Finch House Finch Purple Finch Common Redpoll Red Crossbill Pine Siskin

American Goldfinch Snow Bunting Dark-eyed Junco White-throated Sparrow Rusty Blackbird

\section{.}

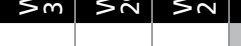

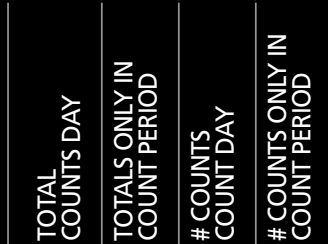

2078

\begin{tabular}{l|l}
5 & 9 \\
0 & 10
\end{tabular}

\begin{tabular}{|l|l|l|l|}
273 & 1 & 6 & 1 \\
\hline
\end{tabular}

\begin{tabular}{|l|l|l|l|}
898 & 21 & 29 & 3 \\
\hline
\end{tabular}

\begin{tabular}{|l|l|l|l|}
56 & 17 & 21 & 6 \\
\hline
\end{tabular}

\begin{tabular}{|l|l|l|l|}
874 & 13 & 41 & 2 \\
\hline
\end{tabular}

\begin{tabular}{|r|r|r|r|r|r|r|r|r|r|r|r|r|r|}
\hline 6 & 14 & & 40 & $(1)$ & & & 86 & & 33 & 2 & 30 & \\
\hline & 1 & & & 1 & & & & & & $(1)$ & & \\
\hline
\end{tabular}

\begin{tabular}{|l|l|l|l|}
\hline 538 & 0 & 57 & 0 \\
\hline
\end{tabular}

\begin{tabular}{|r|r|r|r|r|r|r|r|r|r|r|r|r|}
\hline 1 & & & 1 & & & & & & & & & \\
\hline
\end{tabular}

\begin{tabular}{|l|l|l|l|}
\hline 13 & 1 & 10 & 1 \\
\hline
\end{tabular}

\begin{tabular}{|r|r|r|r|}
5 & 1 & 5 & 1 \\
\hline
\end{tabular}

\begin{tabular}{|r|r|r|r|}
23 & 5 & 15 & 5 \\
\hline
\end{tabular}

\begin{tabular}{|r|r|r|}
\hline 1 & 2 & 11 \\
\hline & 1
\end{tabular}

(1) $\quad 11 \quad 1$
(1)

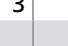

\begin{tabular}{|c|c|c|c|c|c|c|}
\hline & & & & (1) & & 1 \\
\hline & & & & (1) & & \\
\hline 7 & 15 & 3 & & 47 & 2 & 5 \\
\hline 13 & 14 & 1 & 2 & 17 & 2 & 3 \\
\hline 2 & 4 & & 2 & 1 & & \\
\hline
\end{tabular}

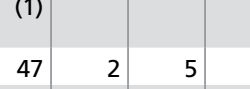

\begin{tabular}{|r|r|r|r|}
\hline & 2 & 1 \\
\hline
\end{tabular}

\begin{tabular}{r|r|r|r|}
\hline 3 & 4 & 14 \\
\hline
\end{tabular}

\begin{tabular}{|r|r|r|}
\hline 72 & 6 & 17 \\
\hline 162 & 5 & 42 \\
\hline
\end{tabular}

\begin{tabular}{|r|r|r|r|}
92 & 1 & 24 & 1 \\
\hline
\end{tabular}

\begin{tabular}{|r|r|r|r|}
3 & 2 & 3 & 2 \\
\hline
\end{tabular}

\begin{tabular}{r|}
10 \\
1
\end{tabular}

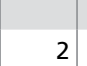

(1)

(1)

(1)

(1)

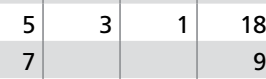

\begin{tabular}{|l|l|r|}
\hline 18 & 1 & 415 \\
\hline
\end{tabular}

\begin{tabular}{|l|l|l|l}
5 & 3 & 4 & 3
\end{tabular}

(2)

\begin{tabular}{|l|l|l|l|}
415 & 0 & 65 & 0 \\
\hline
\end{tabular}

\begin{tabular}{|l|l|l|l|}
\hline 16 & 1 & 61 & 1 \\
\hline 26 & 4 & 15 & 3 \\
\hline
\end{tabular}

\begin{tabular}{r|r|r|r|}
12 & 3 & 8 & 3 \\
\hline
\end{tabular}

\begin{tabular}{|l|l|l|l|}
\hline 6 & 4 & 6 & 3 \\
\hline
\end{tabular}

\begin{tabular}{|l|l|l|l|}
\hline 16 & 2 & 14 & 2 \\
\hline
\end{tabular}

\begin{tabular}{r|r|r|r|}
71 & 0 & 9 & 0 \\
\hline
\end{tabular}

\begin{tabular}{l|l|l}
672 & 2 & 56
\end{tabular}

4492

3849

\begin{tabular}{|l|l|l|l|l|}
24 & 76 & 30 & 4 & 729 \\
\hline
\end{tabular}

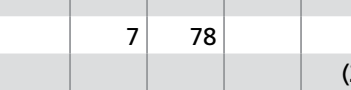


TABLE 4. Species found in fewer than 5 counts.

\begin{tabular}{|c|c|}
\hline SPECIES & LOCALITY AND NUMBER $\left({ }^{*}=\right.$ SEEN DURING COUNT PERIOD) \\
\hline Cackling Goose & Estevan (43), Gardiner Dam (6), Regina (1) \\
\hline Wood Duck & Regina (1) \\
\hline Gadwall & Crooked Lake (1) \\
\hline Northern Pintail & Ft. Qu'Appelle (1*), Grayson (1) \\
\hline Canvasback & Gardiner Dam (4) \\
\hline Greater Scaup & Gardiner Dam (20) \\
\hline Lesser Scaup & Gardiner Dam (16) \\
\hline Bufflehead & Estevan (4), Gardiner Dam (4) \\
\hline Hooded Merganser & Estevan (4), Gardiner Dam (1) \\
\hline Common Merganser & Crooked Lake (2) \\
\hline Red-breasted Merganser & Gardiner Dam (1), Round Lake (1) \\
\hline Ruddy Duck & Estevan (1) \\
\hline Spruce Grouse & E. B. Campbell Dam (1) \\
\hline Pied-billed Grebe & Round Lake (1) \\
\hline Mourning Dove & Avonlea (1), Duval (1), Odessa (2), Weyburn (1) \\
\hline American Coot & Estevan (11) \\
\hline Glaucous Gull & Gardiner Dam (1) \\
\hline Pacific Loon & Gardiner Dam (1) \\
\hline Double-crested Cormorant & Gardiner Dam (1) \\
\hline Northern Harrier & Craven (1) \\
\hline Cooper's Hawk & Estevan (1) \\
\hline Red-tailed Hawk & Kyle $(1 *)$, Odessa (1) \\
\hline Rough-legged Hawk & Balgonie (1), Craven (1), Estuary (1), Qu'Appelle Dam (1) \\
\hline Northern Hawk Owl & Candle Lake (1), E. B. Campbell Dam (1) \\
\hline Great Gray Owl & Shell Lake (1) \\
\hline Long-eared Owl & Kyle (2), White Bear(1) \\
\hline Short-eared Owl & Estevan $(1 *)$, Saskatchewan Landing $\left(3^{*}\right)$, White Bear $(1)$ \\
\hline American Kestrel & Odessa (1) \\
\hline American Crow & Moose Jaw (1), Pike Lake (2), Saskatoon (4) \\
\hline Townsend's Solitaire & $\begin{array}{l}\text { Moose Jaw (2), Qu'Appelle (1*), Saskatoon (1), } \\
\text { Swift Current (2) }\end{array}$ \\
\hline Varied Thrush & Fort Qu'Appelle (1), Indian Head (1), Odessa (2) \\
\hline Hoary Redpoll & E. B. Campbell Dam (2), La Ronge (1) \\
\hline White-winged Crossbill & E. B. Campbell Dam (1), Saskatoon (6) \\
\hline Lapland Longspur & Round Lake (5), Weyburn (77) \\
\hline American Tree Sparrow & Craven (1), Eastend (1), Kyle (3) \\
\hline Fox Sparrow & Grayson (1), Love-Torch River (1), Qu'Appelle Dam (1) \\
\hline White-crowned Sparrow & Archerwill (1) \\
\hline Harris's Sparrow & Whitewood (1) \\
\hline Song Sparrow & Archerwill (1), Cypress Hill P.P. (1) \\
\hline Western Meadowlark & Estevan $(1 *)$ \\
\hline Red-winged Blackbird & Morse (3), Regina (1), Saltcoats (1) \\
\hline Brewer's Blackbird & Qu'Appelle Dam (1) \\
\hline Common Grackle & Avonlea (1), Balgonie (1), Swift Current (1) \\
\hline Northern Cardinal & Prince Albert (1) \\
\hline
\end{tabular}

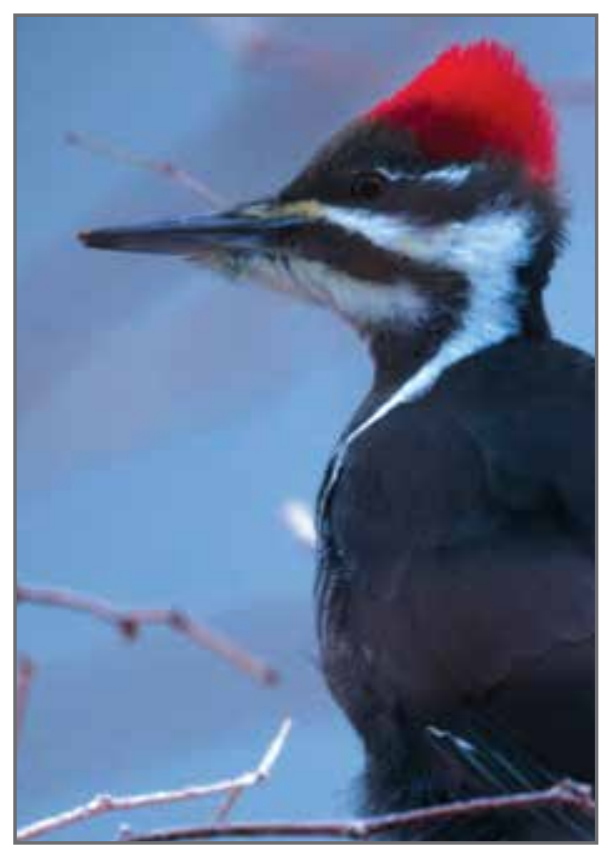

A female Pileated Woodpecker that was observed on the Regina CBC on December 28, 2019. Photo credit: Brian Sterenberg.

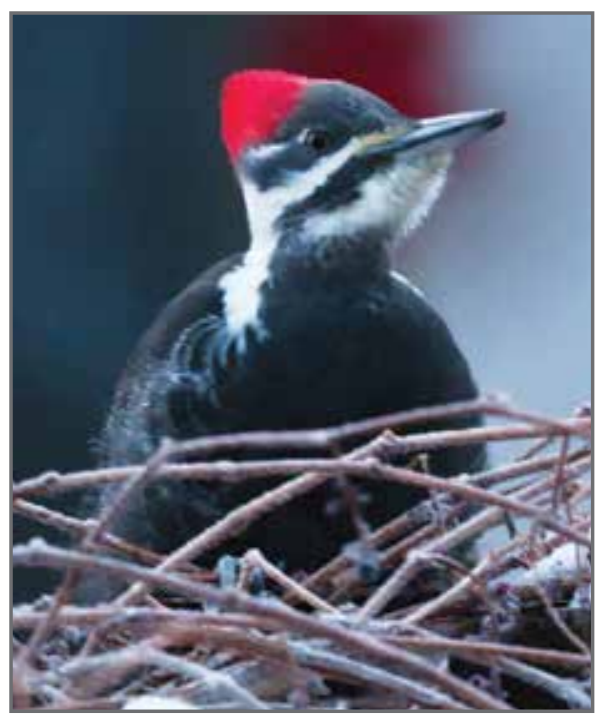

TABLE 5. Birds not identified to species.

\begin{tabular}{|l|l|}
\hline CATEGORY & $\begin{array}{l}\text { LOCALITY AND NUMBER } \\
(*=\text { SEEN DURING } \\
\text { COUNT PERIOD) }\end{array}$ \\
\hline Owl sp. & Regina (1) \\
\hline
\end{tabular}

TABLE 6. New (in bold and italics) and tying high counts for individual species 2019.

\begin{tabular}{|l|l|l|l|l|}
\hline LOCATION & 2019 COUNT & SPECIES & $\begin{array}{l}\text { PREVIOUS } \\
\text { HIGH }\end{array}$ & LOCATION, YEAR \\
\hline $\begin{array}{l}\text { E.B. Campbell } \\
\text { Dam }\end{array}$ & $\mathbf{1 0 7 6}$ & Common Goldeneye & $\mathbf{1 0 5 0}$ & Saskatoon 2016 \\
$\begin{array}{l}\text { Estevan } \\
\begin{array}{l}\text { Gardiner Dam } \\
\text { Gardiner Dam }\end{array}\end{array}$ & $\mathbf{1 3 7 7 5}$ & Canada Goose & $\mathbf{3 0 7 0 0}$ & Coronach (19 December 2001) \\
\hline
\end{tabular}

